\title{
Temozolomide therapy for resistant prolactin-secreting pituitary adenomas and carcinomas: a systematic review
}

\author{
Mussa H. Almalki, ${ }^{1,2}$ Nora Nasser Aljoaib, ${ }^{3}$ Maha Jurais Alotaibi, ${ }^{3}$ Bayan Saloum Aldabas, ${ }^{3}$ \\ Tayba Saleh Wahedi, ${ }^{1}$ Maswood M. Ahmad, ${ }^{1}$ Fahad Alshahrani ${ }^{4}$
}

${ }^{1}$ Obesity, Endocrine, and Metabolism Center, King Fahad Medical City, Riyadh, Saudi Arabia; ${ }^{2}$ King Fahad Medical City, College of Medicine, King Saud bin Abdulaziz University for Health Science, Riyadh, Saudi Arabia; ${ }^{3}$ Almareefa College for Science and Technology (MCST), Riyadh, Saudi Arabia, ${ }^{4}$ King Abdulaziz Medical City, College of Medicine, King Saud bin Abdulaziz University for Health Science, Riyadh, Saudi Arabia

\begin{abstract}
Pituitary tumors represent $10-15 \%$ of all intracranial tumors; of these, prolactinomas account for $40-50 \%$ of cases. Prolactinomas usually respond well to dopamine agonists (DA) as firstline therapy. However, treatment resistance remains a concern. Temozolomide (TMZ) is an oral alkylating agent that has shown promise in treating aggressive pituitary adenomas and carcinomas that are resistant to other therapies. To date, no control trials have been undertaken and only single case reports of pituitary tumors treated with TMZ have been published. A systematic literature search was conducted for studies reporting the use of TMZ for the treatment of prolactinomas that were resistant to standard therapy. In total, 42 reported cases were identified and included in our analysis: 23 cases of prolactin-secreting adenomas and 19 of prolactin-secreting carcinomas. Prior to TMZ administration, patients had exhibited tumor progression and had previously undergone various treatments including surgery, radiotherapy, and drug therapy. Tumor shrinkage was reported in $76 \%$ of patients. Reduced prolactin levels were observed in $75 \%$ of patients, while normalization of prolactin was reported in $8 \%$. TMZ failure occurred in $\mathbf{2 0 . 6 \%}$ of cases. Most patients exhibited no serious adverse effects. In conclusion, TMZ has potential for the treatment of highly aggressive and resistant prolactin-secreting adenomas and carcinomas, as demonstrated by tumor shrinkage or complete response and normalization of hormone hypersecretion, and exhibits good tolerability and few side effects.
\end{abstract}

Key words: Bromocriptine, Cabergoline, Dopamine agonist, Prolactinoma, Resistance, Temozolomide

\section{INTRODUCTION}

Pituitary tumors represent $10-15 \%$ of all intracraAddress for correspondence:

Mussa Hussain Almalki, Obesity, Endocrine and Metabolism Center, King Fahad Medical City, Riyadh, Kingdom of Saudi Arabia; E-mail:m2malki@yahoo.com

Received:06-03-2017, Accepted:06-07-2017 nial tumors. ${ }^{1}$ Of these, prolactinomas are considered to be the most common type of pituitary gland tumor and account for $40-50 \%$ of cases. ${ }^{2}$ Prolactinomas are classified on a clinical basis by size: they are either macroadenomas, which are equal to or larger than $10 \mathrm{~mm}$, or microadenomas, which are smaller than $10 \mathrm{~mm}$ in diameter. ${ }^{3}$ Giant prolactinomas are a rare 
subtype characterized by a tumor size of more than $4 \mathrm{~cm}$, serum prolactin levels that are higher than $1000 \mathrm{ng} / \mathrm{mL}$, massive extrasellar extensions, and no concomitant growth hormone or ACTH secretion. ${ }^{4}$

As per the World Health Organization 2004 classification, pituitary tumors are classified as typical adenomas, atypical adenomas, or pituitary carcinomas. Atypical adenomas are defined by their invasiveness, increased mitotic activity, excessive p53 immunoreactivity, and an MIB-1 proliferative index greater than $3 \%$. Patients may present with multiple recurrences. Malignant prolactinomas are rare tumors defined by the presence of cerebrospinal, meningeal or distant metastasis. They usually have an MIB-1 proliferative index greater than $12 \%$, a high number of mitoses with nuclear pleomorphic features, and positive 533 immunoreactivity. ${ }^{5}$ Patients with prolactinomas present with typical hyperprolactinemia signs and symptoms such as decreased libido, infertility, impotence, galactorrhea, menstrual disturbances or tumor mass effects. ${ }^{3}$

In contrast to other pituitary tumors, prolactinomas respond well to medical therapy alone as first-line treatment. ${ }^{6}$ In general, therapy consisting of dopamine agonists (DA) such as cabergoline and bromocriptine has a high success rate in normalizing prolactin levels, shrinking tumor size, and restoring gonadal functions. Even though most prolactinomas are benign, their resistance to treatment remains a concern. Medical treatment is considered to have failed if prolactin levels are not normalized and tumor size is not reduced by $50 \%{ }^{6}$

In line with recommendations published in the Endocrine Society Clinical Practice Guidelines, patients who are intolerant or resistant to one or more dopamine agonists normally proceed to surgery, the second-line treatment, and frequently to radiotherapy. ${ }^{7}$ Aggressive atypical pituitary tumors tend to be large, invasive, and hyperangiogenic with high mitotic indices, which makes their treatment difficult. They have a tendency to grow continuously and frequently show resistance to dopamine agonist therapy. ${ }^{6}$ Surgical resection is reserved for non-responsive cases or patients with intolerance to medications because of significant associated morbidity and mortality.

Temozolomide (TZM) is an oral alkylating agent that has shown promise in treating resistant aggressive pituitary adenomas and carcinomas with favorable clinical and radiographic responses. TMZ was first used in 2006 for pituitary carcinomas, and many reports have since documented $\mathrm{TMZ}$ as a valuable treatment option that should be added to treatment regimens for resistant prolactinomas. ${ }^{1,8,9}$

The aim of this study was to review the existing literature to date regarding TMZ use and its efficacy for the treatment of resistant aggressive prolactinomas and prolactin-secreting carcinomas.

\section{METHODS}

A systematic search of literature published between January 2000 and May 2017 was conducted regarding the use of TMZ for the treatment of pituitary tumors, specifically prolactinomas that were resistant to standard therapy. A number of articles were identified from PubMed, Google Scholar, and the Cochrane Library using the following search terms: 'prolactinoma', 'resistant', 'dopamine', 'agonist', 'cabergoline', 'bromocriptine', 'temozolomide', 'radiosurgery', 'surgery', 'radiotherapy', and 'pituitary tumors'.

The articles were limited to those published in the English language and all cases mentioned in the articles were analyzed. All relevant publications were reviewed and repetitive articles were excluded. In addition, the reference lists in the articles were searched for potentially relevant informations. Studies that discussed prolactinomas resistance to bromocriptine or cabergoline and those reporting independent retrospective studies of experimental medical therapies for dopamine agonist-resistant prolactinomas are outlined in this study.

We performed a review of published studies and case reports to understand the efficacy of TMZ in the treatment of dopamine agonist-resistant prolactinomas. In total, 42 reported cases of TMZ treatment for prolactin-secreting adenomas and carcinomas fulfilled the study criteria; 23 cases of resistant prolactin-secreting adenoma were reviewed from six individual case reports $^{10,12,19,20,26,27}$ and six small case series..$^{11,16,18,21,22,28}$ Similarly, nine case reports $1,3,9,13,15,17,23-25$ and four small case series ${ }^{11,14,16,21}$ accounted for 19 cases of prolactin-secreting carcinoma. 
As with any other anticancer drug, response to TMZ therapy should be defined as complete response (CR), partial response (PR), progressive disease (PD), and stable disease (SD), according to RECIST (Response Evaluation criteria in solid tumors) version 2.0. Unfortunately, with the exception of one, ${ }^{16}$ none of the studies fulfilled these criteria, which made conclusive statistical analysis of TMZ efficacy in a studied population difficult.

Given the lack of uniform objective criteria in the reviewed studies, we had to analyze whatever figures were available. Information regarding CR, $\mathrm{PR}$, treatment effects, $\mathrm{SD}$, and objective response were collected to assess clinical outcomes. A good response was considered if magnetic resonance imaging (MRI) showed a reduction in tumor size or tumor stabilization, there was a decrease in hormonal hypersecretion, and an improvement in clinical signs and symptoms was evident. TMZ was considered ineffective if there was any progress in tumor size.

\section{RESULTS}

In our study, we identified 42 reported cases of prolactin-secreting pituitary adenomas and carcinomas that used TMZ as a salvage treatment. TMZ was administered as the last resort therapy for aggressive resistant prolactinomas as well as for pituitary carcinomas that did not respond to standard treatments.

Among the 42 patients there were 23 cases of prolactin-secreting adenoma (19 patients had pure prolactinomas, one had an atypical prolactinoma, two had prolactin-secreting adenomas associated with MEN-1, and one had a mixed GH-/PRL-secreting adenoma) (Table 1) and 19 cases of prolactin-secreting carcinoma (16 patients had pure prolactin-secreting carcinomas, two had mixed GH-/PRL-secreting carcinomas, and one patient had a prolactin-secreting carcinoma associated with MEN-1) (Table 2).

Patient ages ranged from 13 months to 78 years. Twenty-seven cases were males and 14 cases were females; sex was not disclosed for one patient. Prior to TMZ initiation, the recruited patients were already experiencing tumor progression and had previously undergone therapies that included dopamine agonists and somatostatin analogues to which they failed to show significant improvement even with escalating doses. Radiotherapy was also used. Although radiotherapy resulted in a transient improvement, subsequent relapse was common. Surgical approaches such as transsphenoidal surgery and debulking of pituitary carcinomas led to a reduction in tumor size, but the effect was temporary because of tumor regrowth and increased PRL levels. In particular, 21 patients were subjected to pituitary surgery at least once. Moreover, 36 patients were also treated with either fractionated or stereotactic radiotherapy.

Data regarding serum prolactin levels pre- and post-TMZ therapy were available for only $10 \mathrm{pa}-$ tients with resistant prolactin-secreting adenoma and nine patients with prolactin-secreting carcinoma. Similarly, objective data for tumor size reduction were not reported in most of the studies reviewed here. The majority of studies mentioned only partial or complete reduction in tumor size as evidence of TMZ therapy efficacy. The effectiveness of TMZ was limited not only in prolactin-secreting adenoma but also in prolactin-secreting carcinoma.

Out of 34 patients for whom objective figures were available, tumor size reduction of varying extents or partial response was reported in $76.5 \%(25 / 34)$ of patients (15 with resistant prolactin-secreting adenoma and 10 with prolactin-secreting carcinoma). Tumor progression was observed in $20.6 \%$ (7/34) of cases (four carcinomas and three resistant prolactin-secreting adenomas), while no change or tumor stabilization was identified in one patient with resistant prolactinsecreting adenoma, and one patient with carcinoma exhibited complete disappearance of their tumor mass. No tumor size data were available for eight patients. A comparison of the two groups revealed that partial response in tumor size was evident in $79 \%$ of atypical resistant prolactinomas and in $66.67 \%$ of carcinomas. Similarly, progressive disease as reflected by increase in tumor size was observed in $15.8 \%$ and $26.67 \%$ of patients with atypical resistant prolactinomas and carcinomas, respectively. Only one patient with prolactin-secreting carcinoma exhibited a complete response.

Biochemical data were available for only 24 patients; complete normalization of hormone hypersecretion was recorded in $8.33 \%(2 / 24)$ of patients, with a 


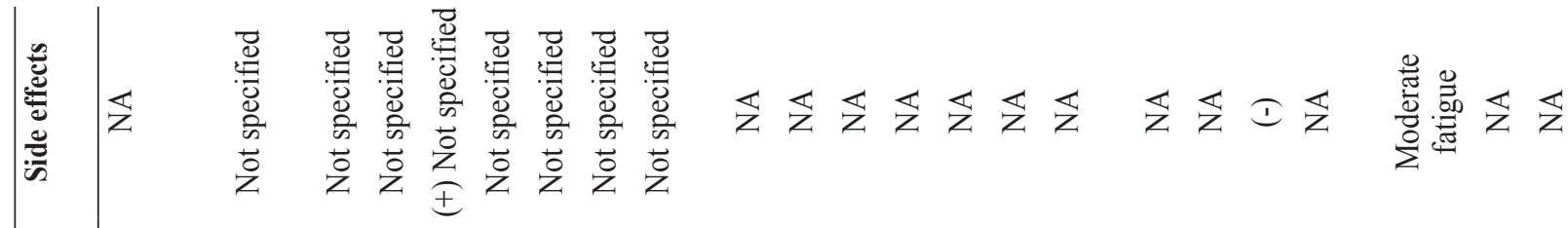
焉

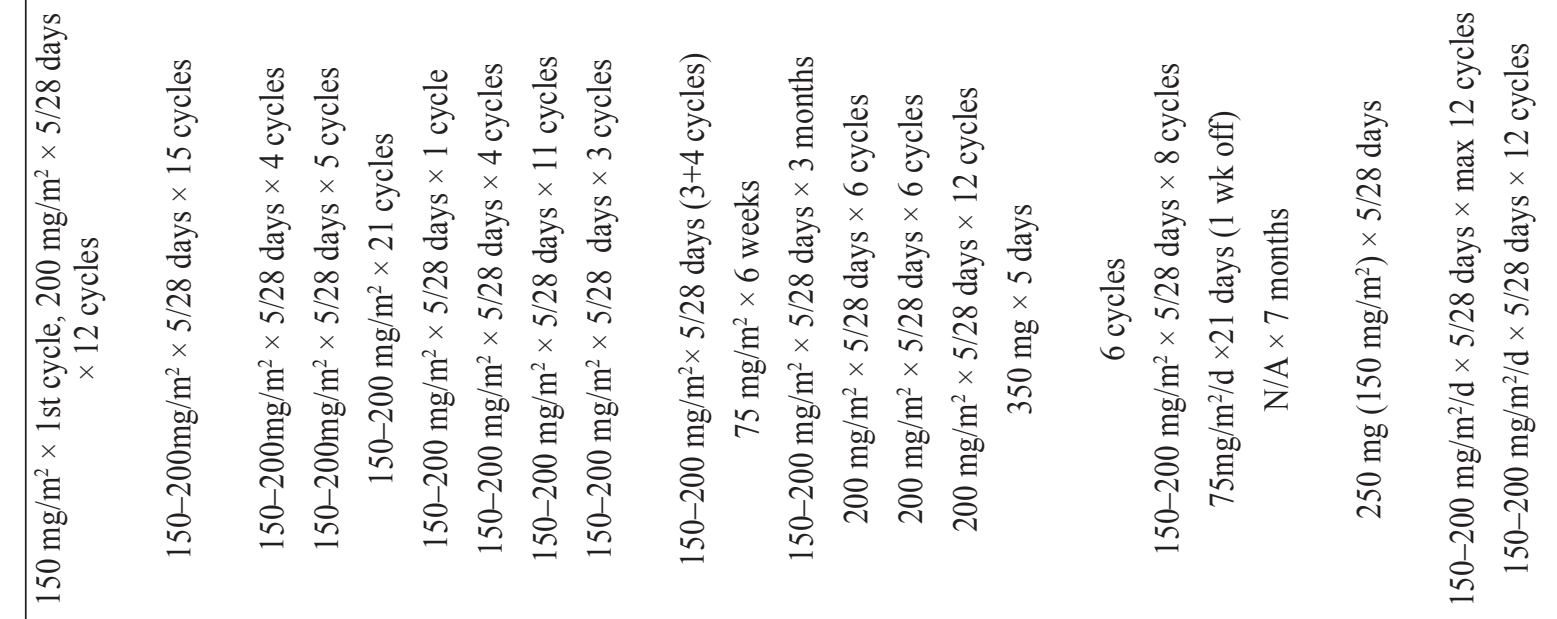

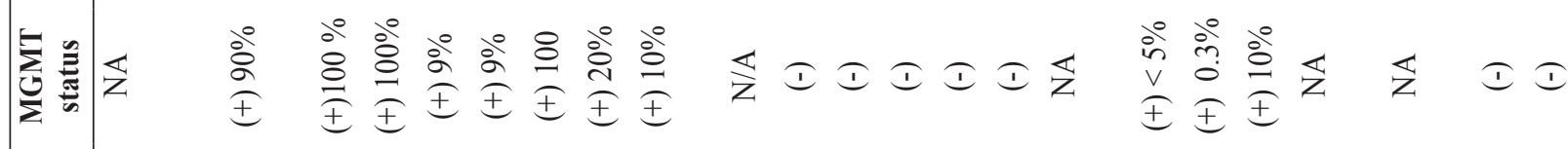

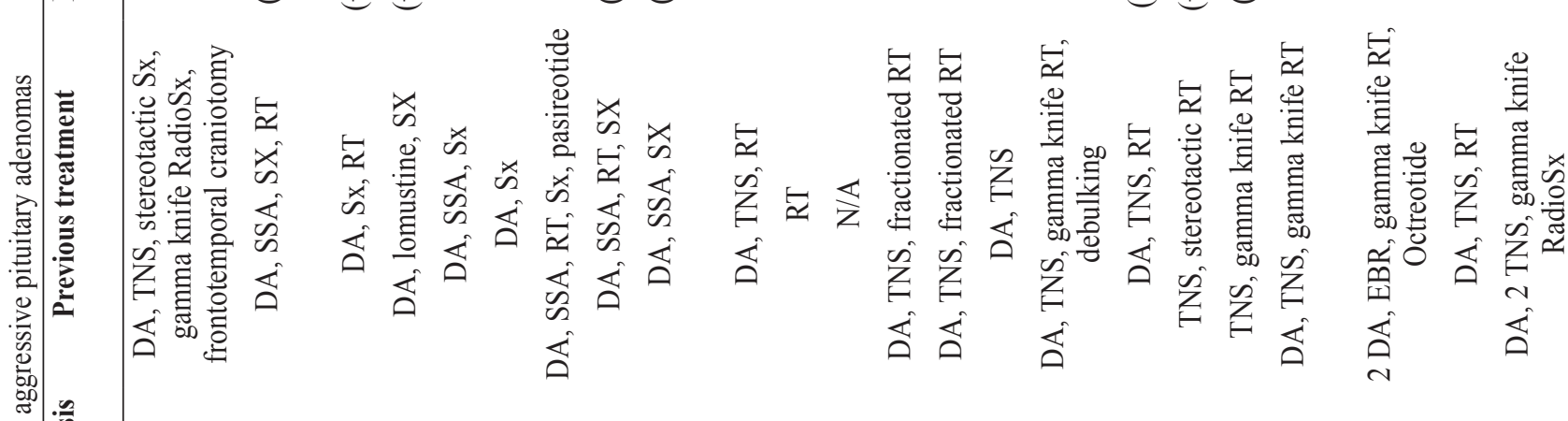

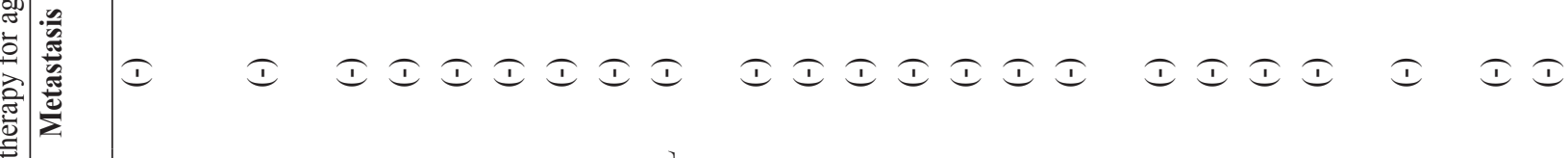

용

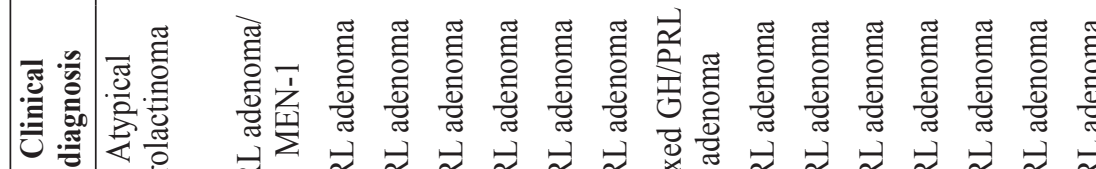

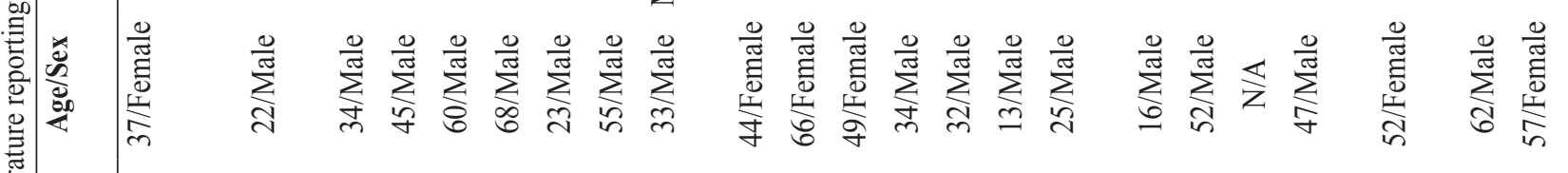

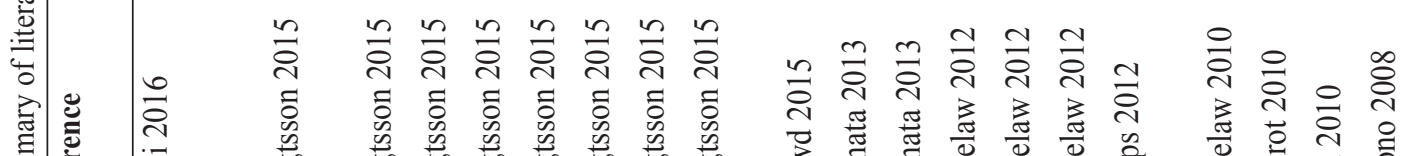

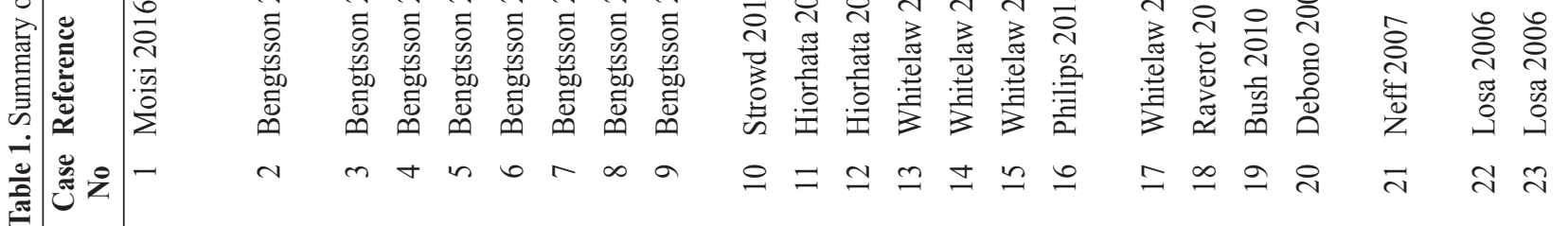




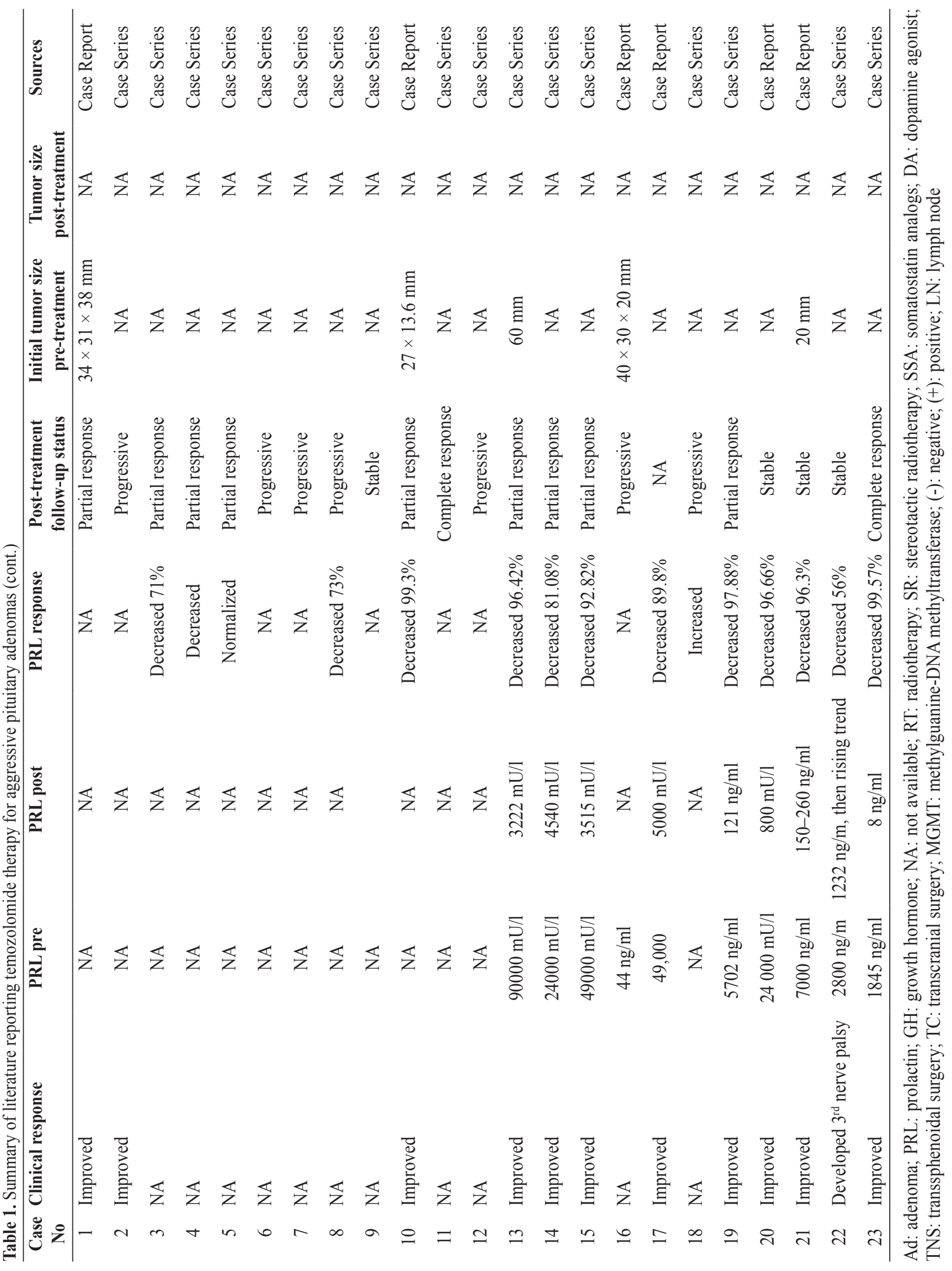




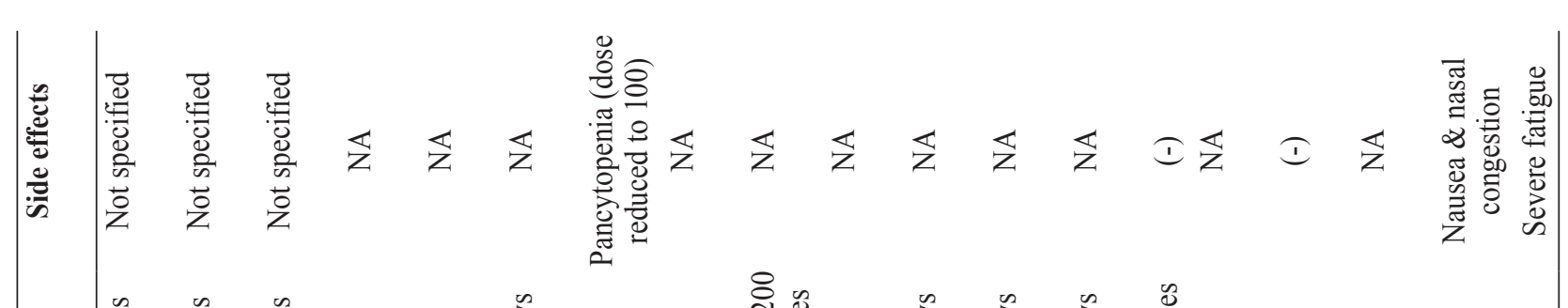

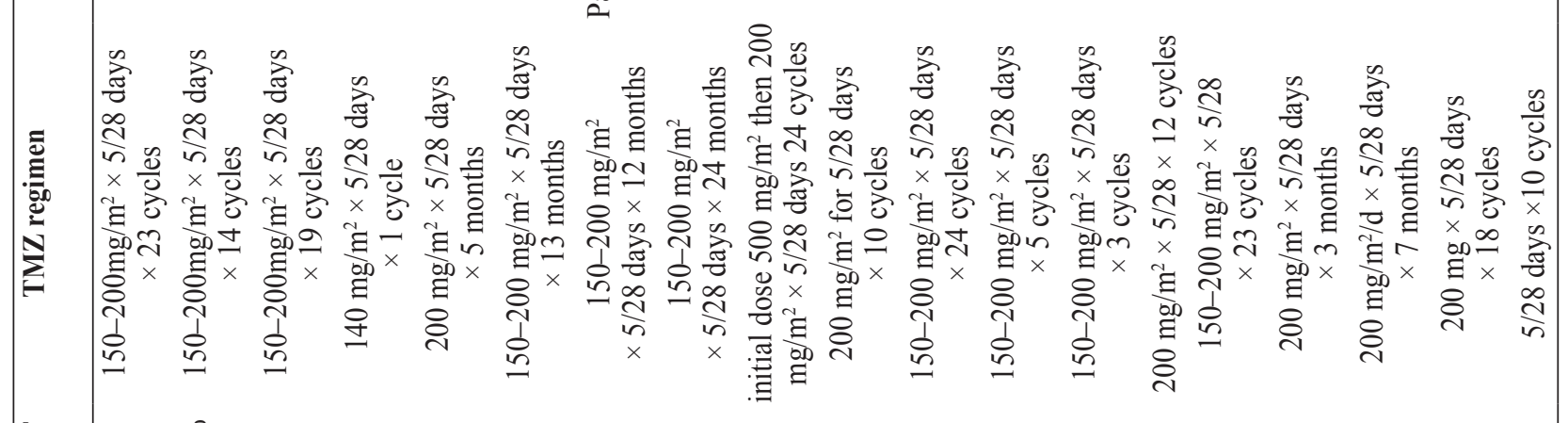

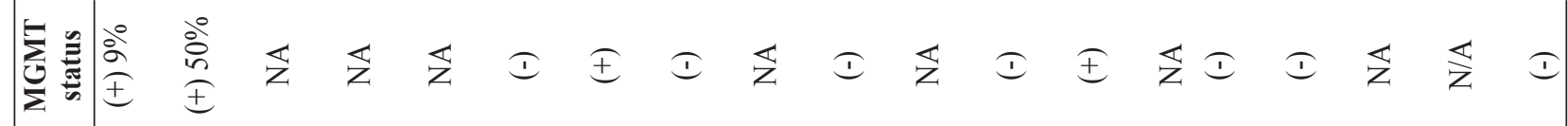

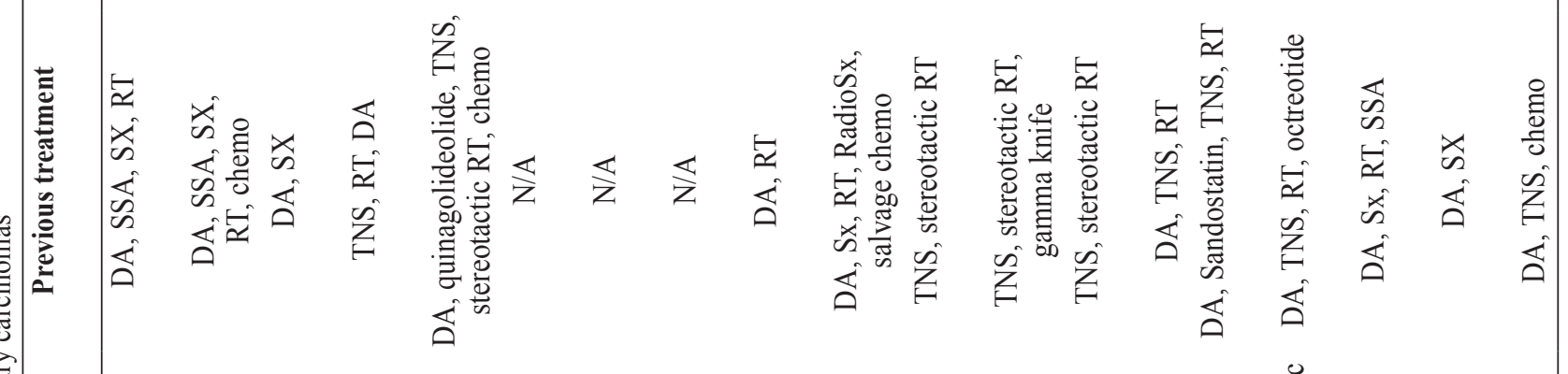

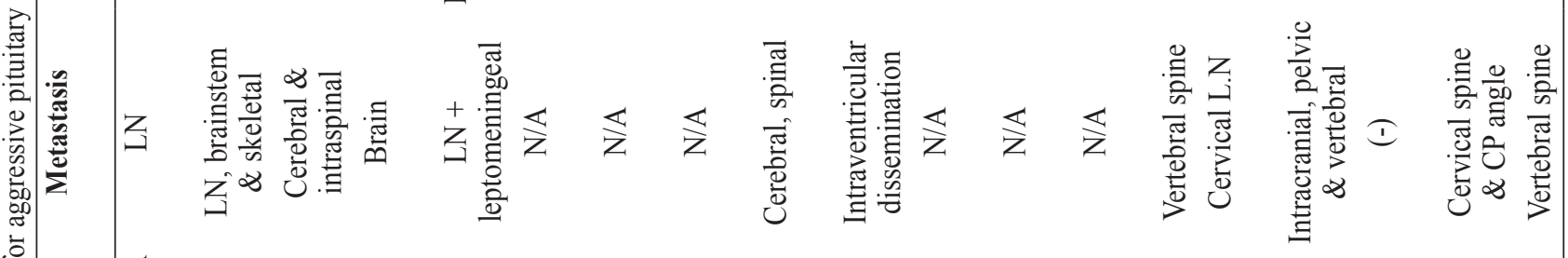

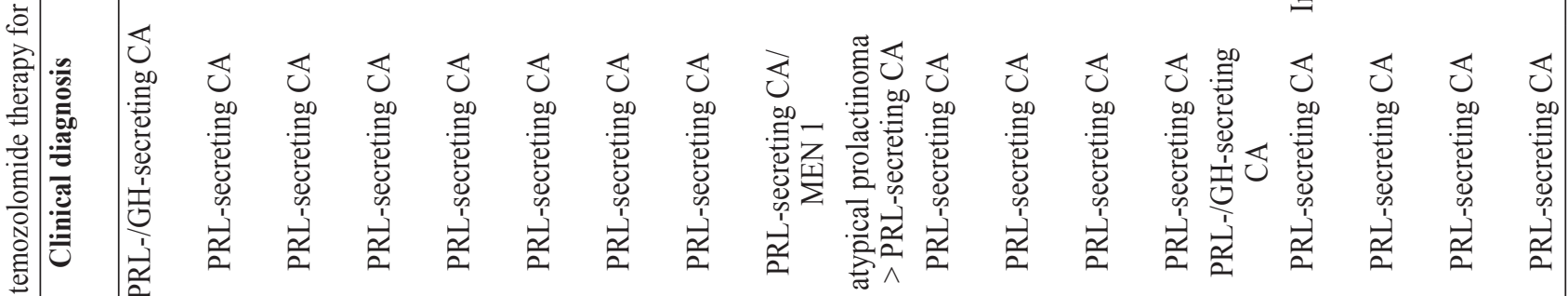

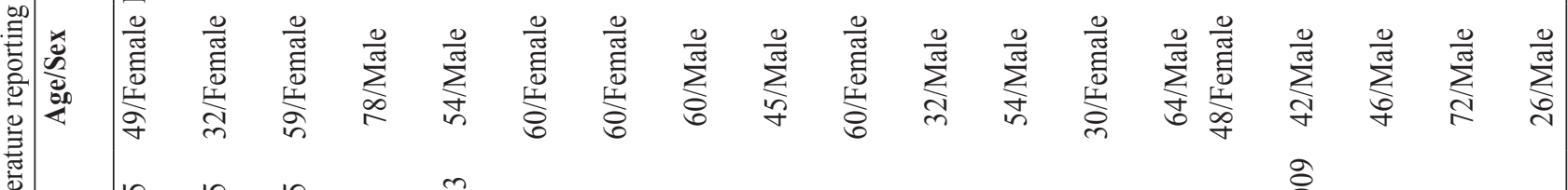

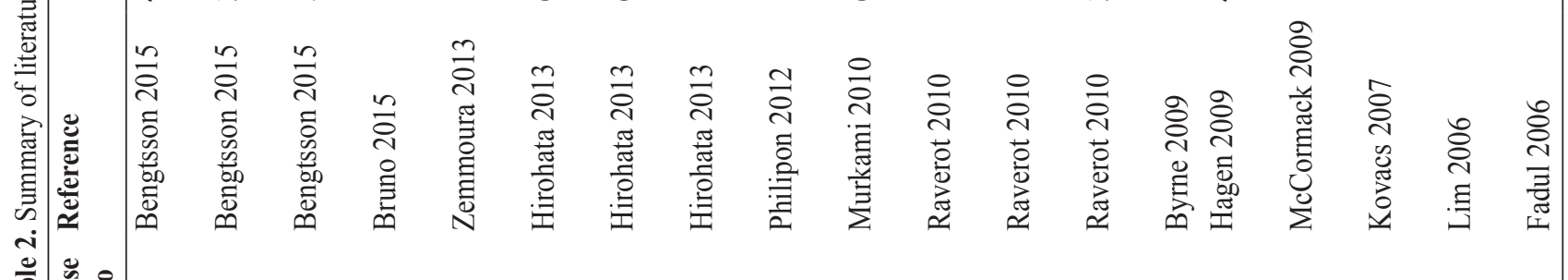

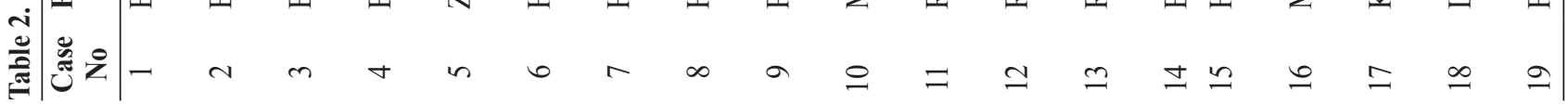




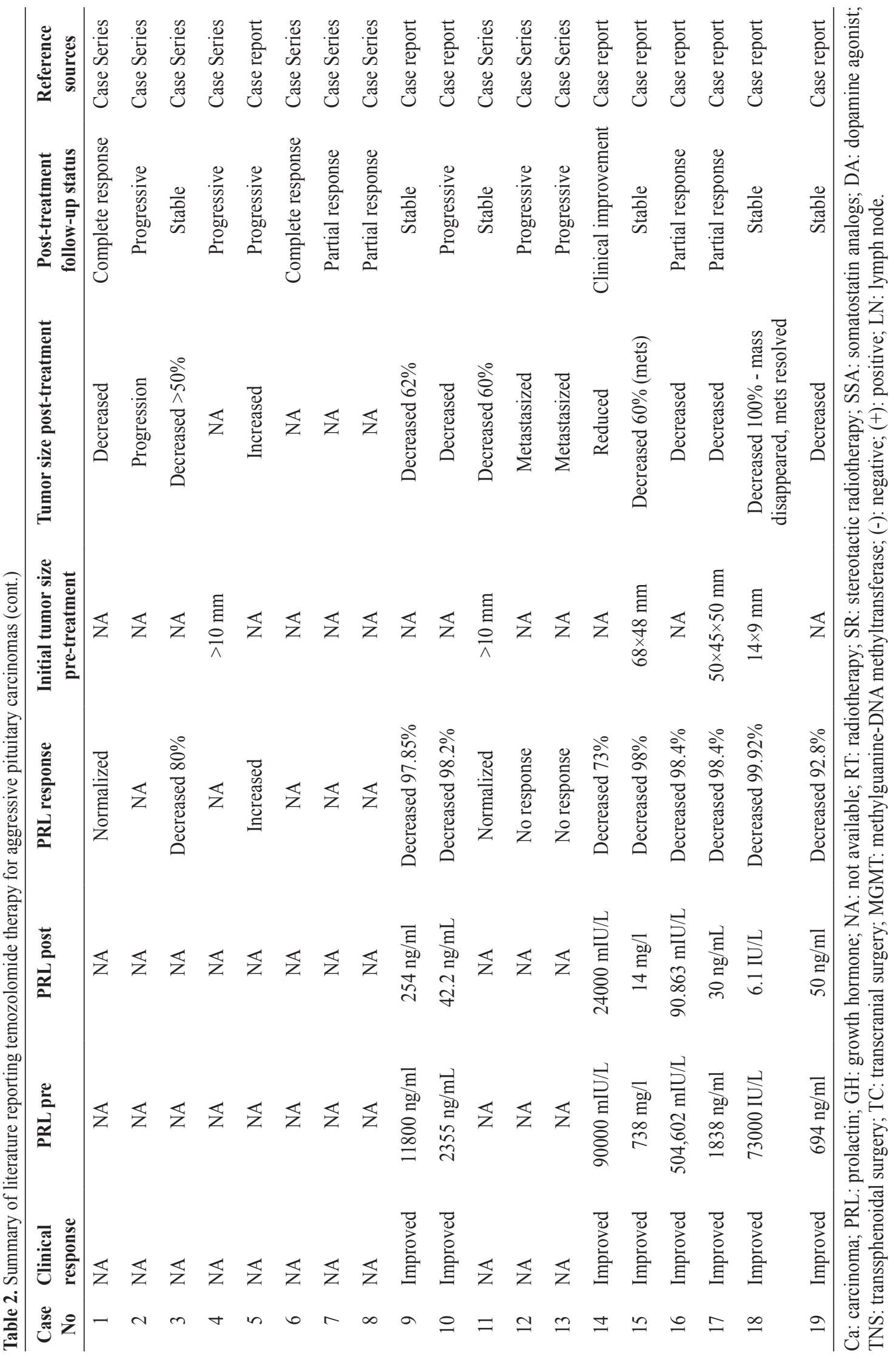


significant decrease in $75 \%(18 / 24)$ of patients. No changes were apparent in $8.33 \%(2 / 24)$ of patients, while progression was observed in another $8.33 \%$ $(2 / 24)$ of cases. A comparison of the two groups revealed that the mean reduction in prolactin level was $89.61 \%$ (56-99.57\%) for resistant prolactin-secreting adenoma and $89.62 \%(50-99.92 \%)$ for prolactinsecreting carcinoma. A cautious interpretation of these values is needed as these data reflected the TMZ efficacy in a very small number of patients and thus the statistical significance is limited.

Death was reported in $29.4 \%(10 / 34)$ of patients who had either no response or who developed tumor progression or even died because of other complications.

Pathological data showed that patients who exhibited negative MGMT expression (14 cases) had an excellent response to TMZ with normalization of PRL and tumor shrinkage. Fifteen patients had varying levels of positive MGMT expression; some experienced disease progression and TMZ treatment failure with no improvement in clinical symptoms and tumor regrowth, while others underwent tumor stabilization.

TMZ was administered orally and the most commonly used therapeutic dose was $150-200 \mathrm{mg} / \mathrm{m}^{2}$ for five of every 28 days. The number of reported treatment cycles ranged from one to 24 cycles.

Most patients experienced no adverse effects, except for a few who complained of fatigability, nausea, nasal congestion, hair loss, headache or dizziness. Other serious but less common side effects were hematological reactions in the form of leucopenia and thrombocytopenia, which were reported in three cases only. Most cases did not require TMZ treatment cessation or dose adjustments, with the exception of one patient whose TMZ treatment was halted after three cycles because of severe hematological toxicity with agranulocytosis.

\section{DISCUSSION}

Even though most prolactinomas initially exhibit a good response to medical therapy alone as first-line treatment, local invasion and recurrence of these tumors makes management of these tumor types challenging. ${ }^{1}$
The challenge presented in treating prolactinomas is not only their resistance to medical treatment but also their poor response to frequent surgical intervention and radiotherapy.

Because of the lack of identification of a consistently effective chemotherapeutic agent, systemic chemotherapy was always reserved as a 'last resort' therapy for prolactinomas.

TMZ, a very old-generation oral alkylating chemotherapeutic agent, is indicated for glioblastoma multiforme, anaplastic astrocytoma, and intracerebral metastasis. ${ }^{29}$ However, recently it has been found to be effective in the treatment of aggressive prolactinomas and has emerged as the first chemotherapeutic to demonstrate substantial efficacy in the treatment of aggressive pituitary tumors. TMZ has few side effects, is available in oral form, and its ability to cross the blood-brain barrier makes it superior to other chemotherapy drugs. ${ }^{28} \mathrm{TMZ}$ acts through methylation of the $\mathrm{O}^{6}$ position of guanine in DNA to form a potent cytotoxic DNA adduct, which causes a disturbance in the DNA sequence, eventually leading to cellular apoptosis. ${ }^{29}$ MGMT is a DNA repair enzyme that reverses alkylation at the $\mathrm{O}^{6}$ position of guanine. This leads to the removal of alkylating adducts and counteracts the pro-apoptotic effect induced by TMZ. A relationship between MGMT status and the effect of TMZ has been proposed. Thus the anti-tumor efficacy of TMZ is limited by high levels of MGMT activity and is thought to offset alkylator modification of tumor DNA. ${ }^{30}$

Another factor affecting TMZ anti-tumor efficacy is the mismatch repair (MMR) pathway, which is involved in the removal of DNA base mismatches caused either by DNA replication errors or through DNA damage. Mutations in the genes involved in the MMR pathway such as MSH2, MLH1, PMS2, and MSH6 have important roles not only in tumor predisposition but also in response to therapy. MMR mediates response to certain forms of DNA damageinducing agents that can modify the structure of bases, including alkylating agents such as TMZ. In MMR-deficient cells, cell cycle and apoptotic responses to these agents are suppressed and cells are chemoresistant. Lack of MSH6 immunopositivity is significantly related to resistance to TMZ therapy with continued tumor growth. ${ }^{31}$ 
The first case of TMZ use for the treatment of resistant pituitary adenomas was reported in $2006 .^{1,9}$ Since then numerous similar small series and case reports have been published, but to our knowledge no systematic review of the literature has been conducted thus far. ${ }^{32}$

Previous case reports suggested that the alkylating agent TMZ was very effective in treating patients with aggressive prolactinomas/carcinomas. ${ }^{28,33} \mathrm{Ap}$ proximately 105 pituitary tumor patients treated with TMZ have been reported in the literature with variable outcomes. ${ }^{14}$ Approximately $60 \%$ of these cases were aggressive adenomas, with the remaining being pituitary carcinomas. The efficacy of TMZ therapy was variable but was generally around $55 \%$ for aggressive adenomas and $58 \%$ for pituitary carcinomas. ${ }^{14}$ The remarkable effect of TMZ was not limited to isolated pituitary tumors, as three cases diagnosed with MEN1 syndrome were reported for which TMZ treatment was also effective..$^{11,17,26}$

In our review, we observed that the cases varied with regard to tumor type, size, invasiveness and malignancy, hormonal secretion, and MGMT status, but in terms of similarity all patients had undergone a trial of medical therapy, radiotherapy, and surgical resection. Although standard therapy temporarily relieved symptoms, their conditions were not resolved. Instead, they deteriorated years later and exhibited worsening symptoms despite conventional multimodal therapy.

In the reported cases, TMZ was introduced when all conventional treatments failed to maintain remission. TMZ was effective even with only one treatment cycle.

It must be highlighted that the criteria used to evaluate response were quite variable and were not as per the strict RECIST criteria. Patient improvement was measured clinically, biochemically, and radiologically but the outcomes were not objectively reported to enable proper statistical analysis.

Our analysis showed a strong association between negative MGMT staining and good response to TMZ. Most cases expressing negative MGMT had an excellent response to TMZ. Among the 42 cases, only 15 patients had positive MGMT expression and TMZ response was disappointing. Death resulted in six of the MGMT-positive cases.
The preferred TMZ regimen is $150-200 \mathrm{mg} / \mathrm{m}^{2}$ for five days of every 28 days $^{32}$ according to the standard scheme used for glioma patients and for neuroendocrine neoplasms. The effects of TMZ on tumors are measured by clinical, radiological, and morphological changes. ${ }^{34}$ Tumor volume reduction and hormonal hypersecretion control were usually observed three months after TMZ initiation. ${ }^{28}$ MRI changes were substantial and included tumor shrinkage, hemorrhage, and necrosis. Morphological changes were studied by Kovac ${ }^{25}$ and Syro, ${ }^{5}$ who compared tumors before and after TMZ administration. Their results showed that following TMZ therapy, tumors were fragile and soft, thus making resection easy; they also reported fewer mitotic cells and low Ki-67 indexes.

Opinions regarding the recommended duration of TMZ therapy vary. ${ }^{8}$ We found that on average 10 14 cycles were effective in generating an excellent response. Although long-term adverse effects were problematic in a small number of cases, TMZ was reported to be very well-tolerated with few side effects, these including nausea, vomiting, and fatigue. Serious events such as myelosuppression were rare. Another issue is prognosis following treatment withdrawal with subsequent resistance, but what is fascinating is that even though TMZ was discontinued, its effectiveness and therapeutic benefits persisted for years. ${ }^{32}$

Predictors of TMZ treatment response have been a subject of debate. Fadul et al ${ }^{1}$ found that patients with pituitary carcinomas who had systemic and CNS metastasis exhibited a good response to TMZ regardless of tumor location or hormonal production status; thus, neither of these factors can be used as treatment predictors for TMZ. MGMT assessment by immunohistochemistry and its inverse relationship with therapeutic response has been proposed by Kovacs et al..$^{25}$ and McCormack et al, ${ }^{24}$ who stated that low MGMT expression conferred a better response to therapy compared with cases of high MGMT expression, which showed resistance.

Whitelaw et al, ${ }^{18}$ suggested early administration of TMZ, which is supported by the fact that most patients presenting late or with complications are left with little time to derive any therapeutic benefit from TMZ therapy.

Tumor recurrence and poor response to chemo- 
therapy in highly aggressive pituitary tumors may justify the early use of TMZ, especially in patients who have already undergone multimodal treatment including surgery and radiotherapy. ${ }^{14}$ Response to TMZ may not be sustained after treatment cessation, but the resumption of TMZ therapy in patients who experience tumor recurrence is possible, as recommended in glioma patients. ${ }^{36}$

TMZ is an excellent choice for treating aggressive prolactin-secreting adenomas and carcinomas, not only for its rapid effects, but also because most patients experienced few or no adverse effects other than mild reactions. The European Society of Endocrinology has also recently endorsed this view in its guidelines. $^{37}$

\section{CONCLUSIONS}

$\mathrm{TMZ}$ is an oral alkylating drug that shows promise for the treatment of highly aggressive resistant pituitary adenomas and carcinomas, as demonstrated by tumor shrinkage or disappearance, as well as normalization of hormone hypersecretion. Indeed, our literature search highlighted the role of TMZ in the treatment of 42 cases, with a success rate reaching $79.5 \%$ for tumor size reduction or stabilization and $83.66 \%$ for biochemical control. This drug not only proved its excellence in controlling clinical symptoms, but also showed substantial radiological and morphological features. Because of its excellent efficacy and good tolerability with few side effects, TMZ can be considered in first-line treatment regimens for highly aggressive prolactin-secreting adenomas and carcinomas, which is in line with the recently published Clinical Practice Guidelines of the European Society of Endocrinology. However, further studies are required to establish appropriate starting doses, maintenance doses, and duration of $\mathrm{TMZ}$ administration for prolactin secreting tumors.

\section{DECLARATION OF INTEREST}

The authors declare that they have no conflict of interest.

\section{FUNDING}

This study was not funded.

\section{REFERENCES}

1. Fadul CE, Kominsky AL, Meyer LP, et al, 2006 Longterm response of pituitary carcinoma to temozolomide. Report of two cases. J Neurosurg 105: 621-626.

2. Gillam MP, Molitch ME, Lombardi G, Colao A, 2006 Advances in the treatment of prolactinomas. Endocr Rev 27: 485-534.

3. Hagen C, Schroeder HD, Hansen S, Hagen C, Andersen M, 2009 Temozolomide treatment of a pituitary carcinoma and two pituitary macroadenomas resistant to conventional therapy. Eur J Endocrinol 161: 631-637.

4. Maiter D, Delgrange E, 2014 Therapy of endocrine disease: the challenges in managing giant prolactinomas. Eur J Endocrinol 170: R213-227.

5. DeLellis RA, Lloyd R, Heitz PU, (eds) 2004 WHO Classification of Tumors, Pathology and Genetics: Tumors of Endocrine Organs. Lyon, France: IARC Press.

6. Oh MC, Aghi MK, 2011 Dopamine agonist-resistant prolactinomas. J Neurosurg 114: 1369-1379.

7. Melmed S, Casanueva FF, Hoffman AR, et al, 2011 Endocrine Society. Diagnosis and treatment of hyperprolactinemia: an Endocrine Society clinical practice guideline. J Clin Endocrinol Metab 96: 273-288.

8. Jaffrain-Rea ML, 2014 From resistant to aggressive and malignant prolactinomas: A translational approach. J Endocr Disord 1: 1012.

9. Lim S, Shahinian H, Maya MM, Yong W, Heaney AP, 2006 Temozolomide: a novel treatment for pituitary carcinoma. Lancet Oncol 7: 518-520.

10. Moisi M, Cruz AS, Benkers T, Rostad S, 2016 Treatment of aggressive prolactin-secreting pituitary adenomas with adjuvant temozolomide chemotherapy: A review. Cureus 8: e658.

11. Bengtsson D, Schrøder HD, Andersen M, et al, 2015 Long-term outcome and MGMT as a predictive marker in 24 patients with atypical pituitary adenomas and pituitary carcinomas given treatment with temozolomide. J Clin Endocrinol Metab 100: 1689-1698.

12. Strowd RE, Salvatori R, Laterra JJ, 2015 Temozolomide retreatment in a recurrent prolactin-secreting pituitary adenoma: Hormonal and radiographic response. J Oncol Pharm Pract 0: 1-6.

13. Murakami M, Mizutani A, Asano S, et al, 2011 A mechanism of acquiring temozolomide resistance during transformation of atypical prolactinoma into prolactin-producing pituitary carcinoma. Neurosurgery 68: 1761-1767.

14. Bruno OD, Juárez-Allen L, Christiansen SB, et al, 2015 Temozolomide therapy for aggressive pituitary tumors: results in a small series of patients from Argentina. Int J Endocrinol 2015: 587-893.

15. Zemmoura I, Wierinckx A, Vasiljevic A, Jan M, Trouillas J, François P, 2013 Aggressive and malignant prolactin pituitary tumors: pathological diagnosis and patient 
management. Pituitary 16: 515-522.

16. Hirohata T, Asano K, Ogawa Y, et al, 2013 DNA mismatch repair protein (MSH6) correlated with the responses of atypical pituitary adenomas and pituitary carcinomas to temozolomide: the national cooperative study by the Japan Society for Hypothalamic and Pituitary Tumors. J Clin Endocrinol Metab 98: 11301136.

17. Philippon M, Morange I, Barrie M, et al, 2012 Longterm control of a MEN1 prolactin secreting pituitary carcinoma after temozolomide treatment. Ann Endocrinol (Paris) 73: 225-229.

18. Whitelaw BC, Dworakowska D, Thomas NW, et al, 2012 Temozolomide in the management of dopamine agonist-resistant prolactinomas. Clin Endocrinol (Oxf) 76: 877-886.

19. Phillips J, East HE, French SE, Melcescu E, et al, 2012 What causes a prolactinoma to be aggressive or to become a pituitary carcinoma? Hormones (Athens) 11: 477-482.

20. Whitelaw BC, Dworakowska D, Thomas NW, et al, 2012 Temozolomide treatment of a resistant prolactinomas. Clin Endocrinol (Oxf) 76: 877-886.

21. Raverot G, Sturm N, de Fraipont F, et al, 2010 Temozolomide treatment in aggressive pituitary tumors and pituitary carcinomas: a French multicenter experience. J Clin Endocrinol Metab 95: 4592-4599.

22. Bush ZM, Longtine JA, Cunningham T, et al, 2010 Temozolomide treatment for aggressive pituitary tumors: correlation of clinical outcome with $\mathrm{O}(6)$-methylguanine methyltransferase (MGMT) promoter methylation and expression. J Clin Endocrinol Metab 95: E280-290.

23. Byrne S, Karapetis C, Vrodos N, 2009 A novel use of temozolomide in a patient with malignant prolactinoma. J Clin Neurosci 16: 1694-1696.

24. McCormack AI, McDonald KL, Gill AJ, et al, 2009 Low O6-methylguanine-DNA methyltransferase (MGMT) expression and response to temozolomide in aggressive pituitary tumours. Clin Endocrinol (Oxf) 71: 226-233.

25. Kovacs K, Horvath E, Syro LV, et al, 2007 Temozolomide therapy in a man with an aggressive prolactinsecreting pituitary neoplasm: Morphological findings. Hum Pathol 38: 185-189.
26. Debono M, Bridgewater C, Ross R, Newell-Price J, 2008 Treating an aggressive prolactinoma in a patient with MEN 1: beneficial response to temozolomide. Endocrine Abstracts 15: P188.

27. Neff LM, Weil M, Cole A, et al, 2007 Temozolomide in the treatment of an invasive prolactinoma resistant to dopamine agonists. Pituitary 10: 81-86.

28. Losa M, Mazza E, Terreni MR, McCormack A, et al, 2010 Salvage therapy with temozolomide in patients with aggressive or metastatic pituitary adenomas: experience in six cases. Eur J Endocrinol 163: 843-851.

29. Knizhnik AV, Roos WP, Nikolova T, et al, 2013 Survival and death strategies in glioma cells: autophagy, senescence and apoptosis triggered by a single type of temozolomide-induced DNA damage. PLoS One 8: e55665.

30. Bello MJ, De Campos JM, Isla A, Casartelli C, Rey JA, 2006 Promoter CpG methylation of multiple genes in pituitary adenomas: frequent involvement of caspase-8. Oncol Rep 15: 443-448.

31. Martin SA, Lord CJ, Ashworth A, 2010 Therapeutic targeting of the DNA mismatch repair pathway. Clin Cancer Res 16: 5107-5113.

32. Mortini P, Barzaghi R, Losa M, Boari N, Giovanelli M, 2007 Surgical treatment of giant pituitary adenomas: strategies and results in a series of 95 consecutive patients. Neurosurgery 60: 993-1004.

33. Liu JK, Patel J, Eloy JA, 2015 The role of temozolomide in the treatment of aggressive pituitary tumors. J Clin Neurosci 22: 923-929.

34. Syro LV, Ortiz LD, Scheithauer BW, et al, 2011 Treatment of pituitary neoplasms with temozolomide: a review. Cancer 117: 454-462.

35. Syro LV, Uribe H, Penagos LC, et al, 2006 Antitumour effects of temozolomide in a man with a large, invasive prolactin-producing pituitary neoplasm. Clin Endocrinol (Oxf) 65: 552-553.

36. Khasraw M, Bell D, Wheeler H, 2009 Long-term use of temozolomide: could you use temozolomide for life in gliomas? J Clin Neurosci 16: 854-855.

37. Raverot G, Burman P, McCormack A, et al, Clinical practice guideline for the management of aggressive pituitary tumors and carcinomas. (In press). 